\title{
A HIGH SIGNAL-TO-NOISE RATIO COMPOSITE QUASAR SPECTRUM ${ }^{1}$
}

\author{
Paul J. Francis and Paul C. Hewett \\ Institute of Astronomy, Madingley Road, Cambridge, CB3 OHA, England \\ Craig B. Foltz and Frederic H. ChafFeE ${ }^{2}$ \\ Multiple Mirror Telescope Observatory, University of Arizona, Tucson, Arizona 85721 \\ AND \\ RAY J. WeYmann AND Simon L. MORRIS \\ Observatories of the Carnegie Institution of Washington, 813 Santa Barbara Street, Pasadena, California 91101 \\ Received 1990 August 27; accepted 1990 November 21
}

\begin{abstract}
We present a very high signal-to-noise ratio $(\mathrm{S} / \mathrm{N} \sim 400)$ composite spectrum of the rest-frame ultraviolet and optical region of high luminosity quasars. The spectrum is derived from 718 individual spectra obtained as part of the Large Bright Quasar Survey. The moderate resolution, $\lesssim 4 \AA$, and high signal-to-noise ratio allow numerous weak emission features to be identified. Of particular note is the large equivalent-width of the Fe II emission in the rest-frame ultraviolet and the blue continuum slope of the composite. The primary aim of this paper is to provide a reference spectrum for use in line identifications, and a series of large-scale representations of the composite spectrum are shown. We also present a measure of the standard deviation of the individual quasar spectra from the composite spectrum.
\end{abstract}

Subject headings: line identifications - quasars

\section{INTRODUCTION}

The first phase of the Large Bright Quasar Survey or LBQS (Foltz et al. 1987, 1989; Hewett et al. 1991) is now complete and the analysis of the spectroscopic properties of this unique sample has begun. We are developing a model-fitting approach to the analysis of the individual quasar spectra in order that parameters describing the rest-frame ultraviolet and optical spectra of the sample can be extracted in an automated, welldefined and homogeneous fashion. We have begun by constructing a "composite" quasar spectrum to identify which components (including emission and absorption features) need to be incorporated in our model-fitting procedure. While two recent papers have presented composite spectra derived from smaller quasar samples (Boyle 1990; Cristiani \& Vio 1990) the quality of the LBQS sample allows us to produce a composite spectrum of unprecedented signal-to-noise ratio (peak continuum $\mathrm{S} / \mathrm{N} \sim 400)$ and relatively high resolution $(2-4 \AA$ in the rest frame of the composite quasar).

Our composite provides a valuable reference particularly for weak emission lines, for the calculation of $k$-corrections for use in studies of the evolution of the quasar population, and for the derivation of the form of the underlying continuum. It also demonstrates the importance of taking into account the extremely strong iron emission when extracting properties of certain emission lines usually thought to lie in "uncontaminated" regions of the spectrum. Section 2 summarizes the characteristics of the LBQS sample. The procedures used to construct the composite are described in $\S 3$, and the main results are presented in graphical and tabular form in $\S 4$. Section 5 includes a brief discussion of the proper-

\footnotetext{
${ }^{1}$ Observations reported here were obtained with the Multiple Mirror Telescope, a facility operated jointly by the University of Arizona and the Smithsonian Institution, and with the Las Campanas Observatory, a facility of the Carnegie Institution of Washington.

${ }^{2}$ Senior Visiting Fellow, Institute of Astronomy, 1990 July 1-1991 Aug 1.
}

ties of the iron emission and the form of the underlying continuum.

\section{THE LBQS QUASAR SAMPLE}

The majority of the quasars were observed at the Multiple Mirror Telescope (MMT) which produced observed-frame spectra covering 3200-7400 $\AA$ at a resolution of $6 \AA$. Some 200 quasars lie in the southern sky and were observed using the $2.5 \mathrm{~m} \mathrm{Du}$ Pont Telescope of the Las Campanas Observatory resulting in a coverage of $3200-6800 \AA$ also at $6 \AA$ resolution. Details of the observing procedures can be found in Foltz et al. (1987) and Morris et al. (1991, in preparation). Each quasar in the LBQS was observed until $S / N=12$ was reached per resolution element in the nearest continuum region to $4800 \AA$ in the observed frame. The resulting large sample of uniform $\mathrm{S} / \mathrm{N}$ spectra ensures that no subset will contribute disproportionately to the composite.

The composite presented here is constructed from the 688 quasars (absolute magnitudes $M_{B_{J}}$ ) $\leq-21.5$ and 30 active galactic nuclei (absolute magnitudes $-21.5<M_{B_{J}} \leq-20.5$ ), which show no strong evidence for the presence of broad absorption-line troughs. A Hubble constant of $H_{0}=100 \mathrm{~km}$ $\mathrm{s}^{-1} \mathrm{Mpc}^{-1}$ and a deceleration parameter, $q_{0}=0.0$ are used throughout the paper.

\section{GENERATING THE COMPOSITE}

\subsection{Redshifts}

The generation of the composite spectrum requires an accurate estimate of the redshift for each quasar, and an iterative procedure to refine the initial redshift estimates (based on the interactive definition of emission-line centroids) was employed. To begin, the quasars were ordered by redshift, and the first quasar was rebinned back to the rest frame, using $2.5 \AA$ bins, and scaled such that it had a median flux of unity. Using the initial redshift estimate, each quasar spectrum in turn was rebinned into $2.5 \AA$ wavelength bins in the rest frame, conserving flux during the transformation. 
A scaling factor was then applied to each subsequent spectrum to ensure that it had a median continuum flux equal to that of the partially complete composite over the wavelength range in common. Spectral regions including (i) strong emission lines, (ii) the Lyman- $\alpha$ forest shortward of $1216 \AA$, (iii) the regions shortward of $4200 \AA$ and longward of $7000 \AA$ in the observed frame, and (iv) regions where the composite was composed of less than eight spectra (after the first 15 spectra had been added), were excluded from the continuum scaling calculation. Then, the quasar spectrum was incorporated into the composite with the flux in each wavelength bin weighted by the corresponding reciprocal of the noise variances for the quasar and the composite. These variances were calculated from the signal and sky background, and the known characteristics of the spectrograph/detector. The regions excluded from the scaling calculation were nonetheless incorporated into the composite. Once the first composite was complete the following sequence was performed five times:

1. Each quasar spectrum was cross-correlated with the composite spectrum to derive an improved redshift estimate.

2. All quasar spectra for which the new redshift estimate differed from the old by $\Delta z>0.015$ were inspected visually to identify the very small fraction of spectra for which there was some ambiguity in the assigned redshift.

3. A new composite was generated using the improved redshifts.

Note that the continuum scaling method does not affect the redshift determinations, as the spectra have a low-order polynomial fit subtracted before the cross correlation. The crosscorrelation technique generates a redshift estimate based on all the features present in the spectrum, with the strong features contributing relatively more than the weak. Differences between individual emission line velocities and the crosscorrelation velocities do not significantly effect the generation of the composite. The success of the cross-correlation procedure is demonstrated by the substantial decrease in the width of narrow emission lines such as the [O III] 4959, $5007 \AA$ doublet compared to the composite derived employing the visually assigned redshifts. In only three cases was there any ambiguity in the redshift assignment, and all of these could be traced either to the pathological nature of the individual spectra or to problems arising from their reduction; these spectra were not included in the final composite. The internal errors in the redshift assignments were estimated by crosscorrelating each individual quasar spectrum with two composites, each made up of half the total number of spectra. The resulting dispersion of the redshift differences was $\sigma_{z} \sim 10^{-3}$.

\subsection{The Continuum}

The generation of the composite was undertaken in such a way as to preserve the underlying continuum slope of the quasar sample as a whole, but note that there is no "correct" way of co-adding spectra that exhibit differences on many different scales. The large-scale shape is very sensitive to the exact procedures employed, and four effects in particular might compromise the composite's large scale flux distribution.

1. Galactic and intrinsic extinction may artificially redden the spectrum. However, all the LBQS fields lie at $|b|>50^{\circ}$, and no attempt has been made to correct for the very small ( $\lesssim 5 \%$ ) steepening of the quasar spectra due to galactic extinction. A $5 \%$ steepening would change the power-law index, $\alpha$, by $-0.06\left(F(v) \propto v^{\alpha}\right)$. Similarly, no corrections have been made for the unknown amounts of intrinsic extinction which may be present in the quasars themselves.

2. The MMT spectra were obtained using a 2.5 arcsecond circular aperture, and while all observations were made at an air mass of less than 1.4 (with the majority at less than 1.2), a significant loss of flux at short wavelengths in the observed frame could result from the effects of differential refraction. This possibility was investigated by generating a composite employing only spectra obtained at air masses of less than 1.2 and using only data with observed wavelengths $\lambda>4200 \AA$. This low air mass composite had a continuum shape indistinguishable from that of the full composite. Consequently, spectra observed at all air masses were used to produce the composite. As a final check, the strengths and dispersions of the lines in the low air mass and full composites were compared and found to be statistically indistinguishable.

3. Our scaling method preserves medium-scale features like the $3000 \AA$ bump, but the contribution of individual quasars to the overall continuum shape of the composite depends on the order of addition. Early in the composite construction, each spectrum can have a large effect on the as yet poorly defined overall continuum shape. Subsequent spectra, when added to the composite, will tend to follow this established large-scale shape. This problem affects only features spanning factors of 2 or more in wavelength.

4. Another potential source of systematic error lies in the fact that the arithmetic mean of spectra with different powerlaw slopes does not in general have a power-law index equal to that of the mean of the constituents. In particular, the mean will tend to be steeper than the mean power-law index would suggest. Employing geometric means at the relatively low signal-to-noise ratios of the spectra is not feasible. However, the problem was largely eliminated by scaling the flux in each spectrum by the square of its associated wavelength before co-addition, making each spectrum approximately "flat."

The systematic error in the large-scale composite shape was estimated by employing geometric and arithmetic algorithms in the summation and also by comparing composites generated by summing the individual spectra in different redshift order. No change was observed in the shape of small-scale features such as the emission lines. A softening of the slope in the ultraviolet, and a very prominent $3000 \AA$ bump were always seen, with the bump strength varying by only $5 \%$. However, the power-law slope, $\alpha$, measured between continuum windows at 1450 and $5050 \AA$ varied by $\pm \sim 0.2$. This scatter in large-scale slope is not unexpected, as the cumulative construction of the composite requires only a $0.2 \%$ systematic mis-scaling as each spectrum is added to produce a 0.1 change in the power-law index of the composite.

To ensure that the composite has a large-scale slope representative of the individual component objects, we fitted a simple model continuum to the individual spectra. The model consisted of a power-law and four $\mathrm{Fe}$ II/Balmer continuum components, (as described in $\S 4$ ), which were allowed to vary in intensity only. Although a power-law is an adequate representation of the limited amount of continuum seen in any individual object, the inclusion of four Fe II components, greatly reduces the systematic changes in slope across the $3000 \AA$ bump. Our continuum model was fitted to many strong-linefree regions of the spectra, by minimizing the sum of the deviations, a procedure less affected by outlying points than a least-squares fit. There remains a small systematic trend in the derived power-law slope with redshift $(\Delta \alpha \lesssim 0.3$ peak) which is 


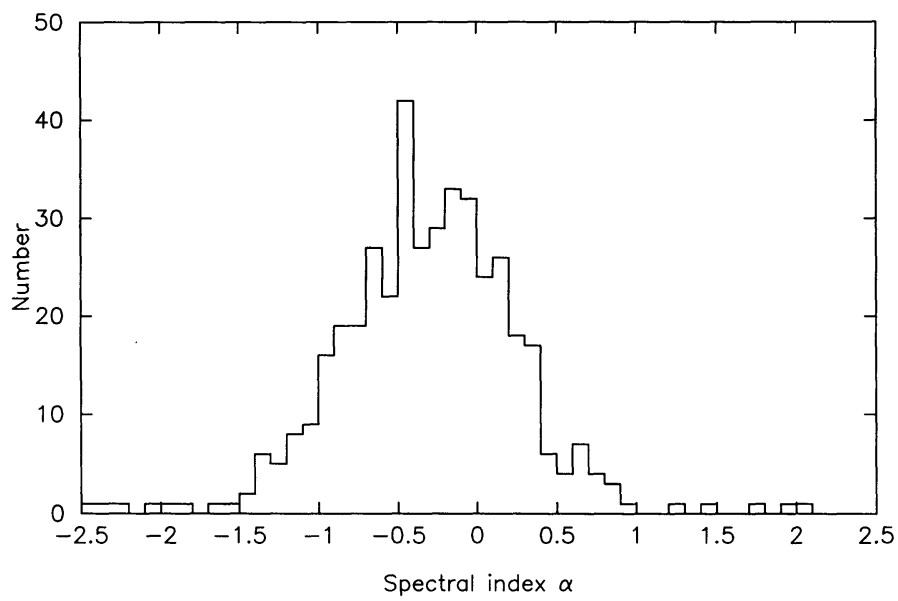

FIG. 1.-Histogram of continuum spectral indices, $\alpha$, where $F(v) \propto v^{\alpha}$, for all quasars and AGNs contributing to the composite spectrum.

partially an artifact of: (1) the lack of continuum windows shortward of $1400 \AA,(2)$ the crudeness of the Fe II model used, and (3) the softening of the continuum shortward of $2000 \AA$. The full range of slopes, as displayed in Figure 1, is, however, present at all redshifts, and changes in the continuum model do not seriously affect this histogram. The median slope is -0.32 , and the final composite was chosen to have this slope, measured between the reddest and bluest continuum windows, at 1450 and $5050 \AA$.

\section{THE COMPOSITE SPECTRUM}

Figure 2 shows the composite spectrum plotted as $\lambda F(\lambda)$ versus $\lambda$ with the principal emission features marked. Figure 3 gives the number of individual spectra which contribute to the composite as a function of wavelength.

The noise variances associated with the individual spectra have been propagated through the generation of the composite to give the noise as a function of wavelength shown in Figure 4 -note the different scale on the $y$-axis compared to Figure 2. In addition, the standard deviation of the fluxes of the individual quasar spectra about the composite is shown in Figure 5. We have assumed that the noise variances associated with each spectrum are independent of the intrinsic variation in the spec-

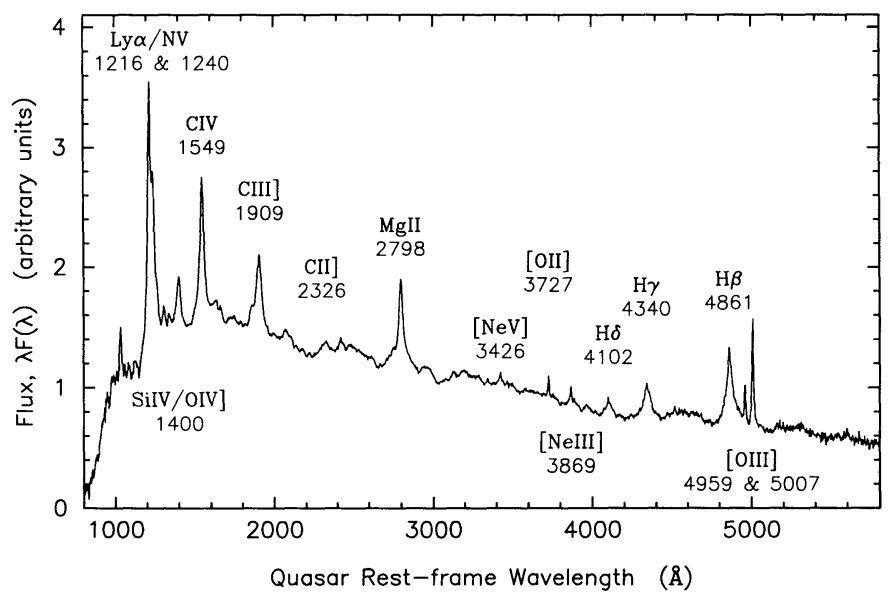

FIG. 2.-Composite spectrum plotted as $\lambda F(\lambda)$ vs. rest-frame wavelength with the principal emission features identified. The flux scale is in arbitrary units.

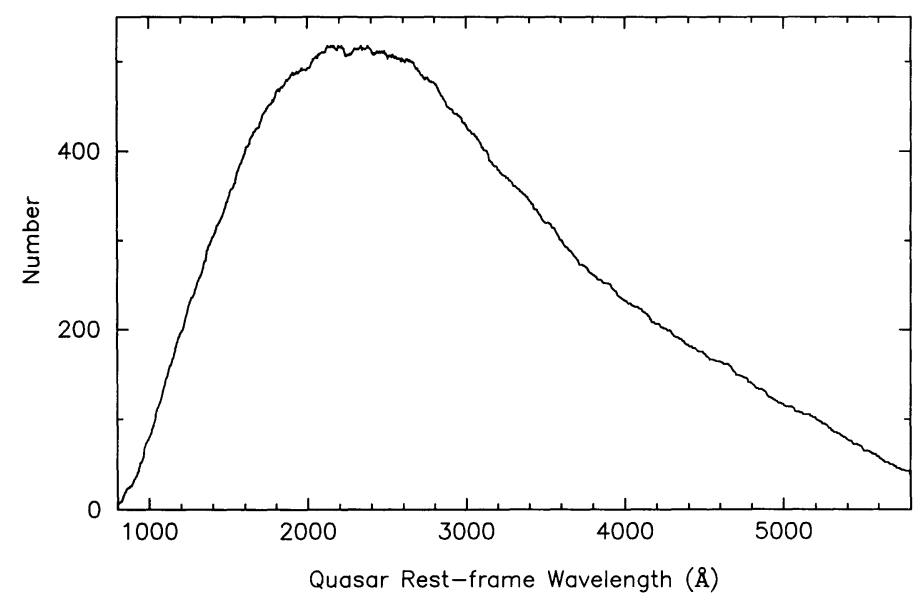

FIG. 3.-Number of quasars and AGNs contributing to the composite as a function of rest-frame wavelength.

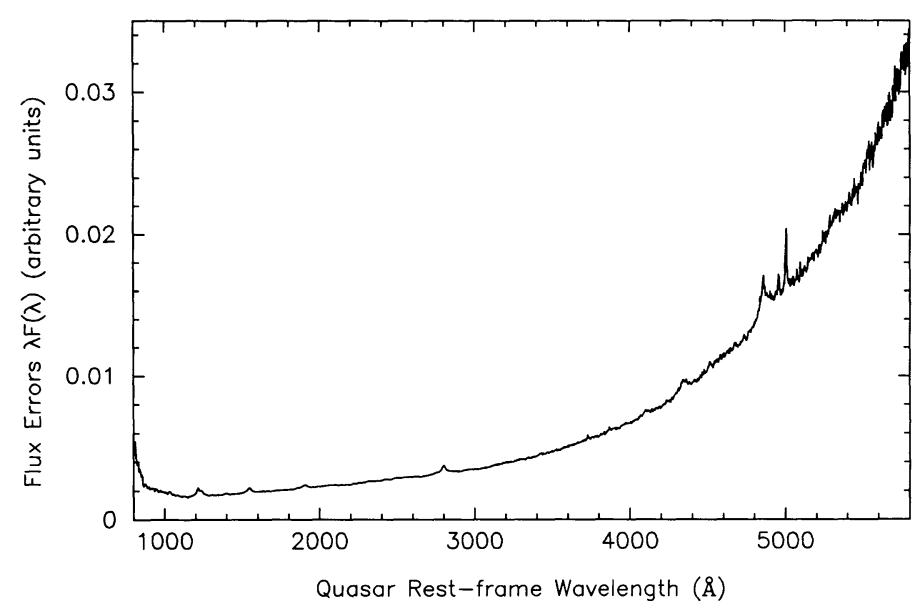

Fig. 4.-Companion error array for the composite spectrum shown in Fig. 2. Note the expanded $y$-scale.

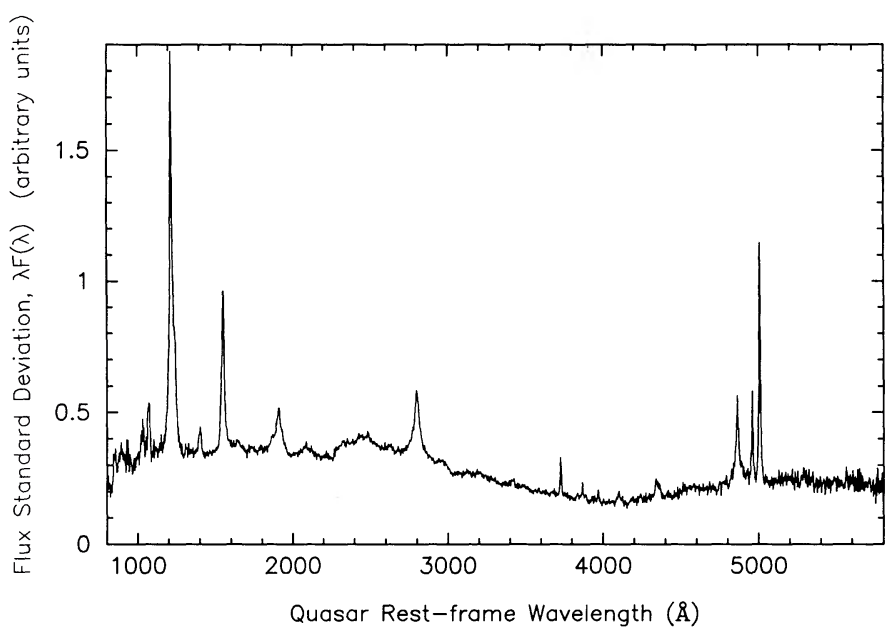

FIG. 5.-Standard deviation in flux relative to the composite spectrum as a function of wavelength for the individual quasars and AGNs making up the composite. This provides a measure of the dispersion of the spectral features about the "mean" values in the composite spectrum. 
tral properties, and Figure 5 shows the standard deviations after subtraction of the noise component. Interpretation of the variations of quasar properties implicit in this figure will be discussed in a future paper (Francis et al. 1991, in preparation).

It is difficult to interpret the continuum dispersion shown in Figure 5, as the large-scale form depends on the procedure employed to generate the composite, and we do not ascribe any significance to the apparent minimum at $\sim 4000 \AA$. We also note that there is no significant increase in the continuum dispersion shortward of the Lyman- $\alpha$ emission line, indicating that fluctations caused by uncorrected Lyman- $\alpha$ forest absorption are not a major contributor to that dispersion at the resolution of our spectra. However, the plot provides some insight into the variation of the individual emission features as well as the blends of $\mathrm{Fe}$ II emission - note the prominent sharp jump at $\sim 2250 \AA$ for example, demonstrating the variability of this often-conspicuous feature.

Table 1 gives details of all emission features that we have been able to identify. Column (1) gives the feature identification and column (2) the wavelength of the proposed identification when relevant. Columns (3) and (4) contain the start and end points over which the flux and equivalent width of the feature were calculated. Relative fluxes, with the Lyman- $\alpha+$ NV blend arbitrarily defined as 100 , are tabulated in column (5) with the associated standard deviation for the stronger lines in column (6). The standard deviations for the fluxes have been calculated using the standard deviations shown in Figure 5 and assuming that the variation in emission features and the continuum are independent. Line equivalent widths are listed in column (7) and references to the table footnotes are given in column (8). As

TABLE 1

LINE STRENGTHS

\begin{tabular}{|c|c|c|c|c|c|c|c|}
\hline Identification & $\begin{array}{l}\text { Restframe } \\
\text { Wavelength } \\
\qquad(\AA)\end{array}$ & $\begin{array}{c}\text { Start }^{2} \\
(\AA)\end{array}$ & $\begin{array}{r}\operatorname{End}^{\mathrm{a}} \\
(\AA)\end{array}$ & $\begin{array}{l}\text { Relative } \\
\text { Flux }{ }^{b}\end{array}$ & $\begin{array}{l}\text { Standard } \\
\text { Deviation }\end{array}$ & $\begin{array}{l}\text { Equivalent } \\
\text { Width } \\
(\AA)\end{array}$ & Note \\
\hline $\mathrm{Ly} \beta+\mathrm{O}$ VI & $1026 \& 1034$ & 1018 & 1054 & 9.3 & $\ldots$ & 5.3 & \\
\hline Ly $\alpha+N$ V & $1216 \& 1240$ & 1186 & 1286 & 100 & 88 & 52 & \\
\hline O I & 1302 & 1288 & 1325 & 3.5 & $\ldots$ & 1.9 & \\
\hline C II & 1335 & 1325 & 1354 & 2.5 & $\ldots$ & 1.3 & \\
\hline Si IV + O IV] & 1400 & 1353 & 1454 & 19 & 5 & 10 & \\
\hline C IV & 1549 & 1452 & 1602 & 63 & 41 & 37 & \\
\hline $\mathrm{He}$ II + O III] & $1640 \& 1663$ & 1602 & 1700 & 18 & 21 & 12 & (1) \\
\hline $\mathrm{Al}$ III + C III] & $1858 \& \quad \& \quad 1909$ & 1828 & 1976 & 29 & 25 & 22 & \\
\hline 2000 feature & $\ldots$ & 1985 & 2018 & 0.49 & $\ldots$ & 0.42 & \\
\hline 2080 feature & $\ldots$ & 2035 & 2125 & 4.1 & $\ldots$ & 3.7 & (2) \\
\hline 2140 feature & $\ldots$ & 2125 & 2158 & 0.34 & $\ldots$ & 0.32 & (3) \\
\hline 2175 feature & $\ldots$ & 2158 & 2204 & 0.76 & $\ldots$ & 0.78 & (4) \\
\hline 2200 dip? & $\ldots$ & $\ldots$ & $\ldots$ & $\ldots$ & $\ldots$ & & (5) \\
\hline 2225 feature & $\ldots$ & 2206 & 2238 & 0.47 & $\ldots$ & 0.51 & \\
\hline C II] & 2326 & 2242 & 2388 & 6.0 & $\ldots$ & 6.4 & \\
\hline [Ne IV] & 2423 & 2386 & 2464 & 2.2 & $\ldots$ & 2.39 & (6) \\
\hline $\mathrm{Mg}$ II & 2798 & 2650 & 2916 & 34 & 20 & 50 & (7) \\
\hline 2970 feature & $\ldots$ & 2908 & 3026 & 6.3 & $\ldots$ & 10 & (8) \\
\hline 3130 feature & $\ldots$ & 3100 & 3156 & 0.73 & $\ldots$ & 1.3 & \\
\hline 3200 feature & $\ldots$ & 3156 & 3236 & 0.95 & $\ldots$ & 1.7 & (9) \\
\hline$[\mathrm{Ne} \mathrm{V}]$ & 3346 & 3324 & 3372 & 0.52 & $\ldots$ & 1.0 & \\
\hline$[\mathrm{Ne} \mathrm{V}]$ & 3426 & 3392 & 3452 & 1.0 & $\ldots$ & 2.1 & \\
\hline$[\mathrm{O} \mathrm{II}]$ & 3727 & 3712 & 3742 & 0.78 & 1.5 & 1.9 & \\
\hline$[\mathrm{Ne} \mathrm{III}]+\mathrm{He} \mathrm{I}$ & $3869 \quad \& \quad 3889$ & 3804 & 3934 & 3.6 & $\ldots$ & 9.8 & (10) \\
\hline [Ne III] & 3968 & 3934 & 4012 & 1.3 & $\ldots$ & 3.9 & \\
\hline$[\mathrm{S} \mathrm{II}]+\mathrm{H} \delta$ & $4068 / 4076 \quad \& \quad 4102$ & 4044 & 4148 & 2.8 & $\ldots$ & 8.9 & \\
\hline $\mathrm{H} \gamma+[\mathrm{O} \mathrm{III}]$ & $4340 \& 4363$ & 4276 & 4405 & 13 & 3.3 & 9.8 & \\
\hline $\mathbf{H} \beta$ & 4861 & 4704 & 5112 & 22 & 4.1 & 58 & \\
\hline [O III] & 4959 & 4942 & 4976 & 0.93 & 1.5 & 3.8 & \\
\hline [O III] & 5007 & 4986 & 5044 & 3.4 & 3.6 & 15 & \\
\hline \multicolumn{8}{|c|}{ Fe II COMPONENTS:- } \\
\hline 1 & $\ldots$ & 1610 & 2210 & 46 & 18 & & \\
\hline 2 & $\ldots$ & 2210 & 2730 & 26 & 69 & & \\
\hline 3 & $\ldots$ & 2960 & 4040 & 39 & 23 & & (11) \\
\hline 4 & $\ldots$ & 4340 & 4830 & 11 & 8 & & \\
\hline 5 & $\ldots$ & 5050 & 5520 & 6.8 & $\ldots$ & & \\
\hline
\end{tabular}

${ }^{a}$ Wavelength limits between which the line flux was integrated.

b Percent of combined flux of $\mathrm{Ly} \alpha+\mathrm{N} v$.

NoTES.- (1) Separation from C IV arbitrary; (2) Possible contribution from Fe II; (3) Possible contribution from $\mathrm{N}$ II $\lambda 2140$; (4) Possible contribution from He II $\lambda 2186$, but note that $\mathrm{He}$ II $\lambda 4686$ is not seen; (5) Silicate dust absorption feature or gap in the Fe II emission?; (6) Flux and equivalent width calculated after a $20 \%$ correction for second-order Ly $\alpha$ contamination; (7) Blended with Fe II emission; (8) Possible contribution from Fe II; (9) Possible contribution from $\mathrm{He}$ II $\lambda 3203$, but note absence of $\mathrm{He}$ II $\lambda 4686$; (10) Continuum fitting particularly uncertain due to Balmer emission; (11) This component includes the Balmer continuum. 


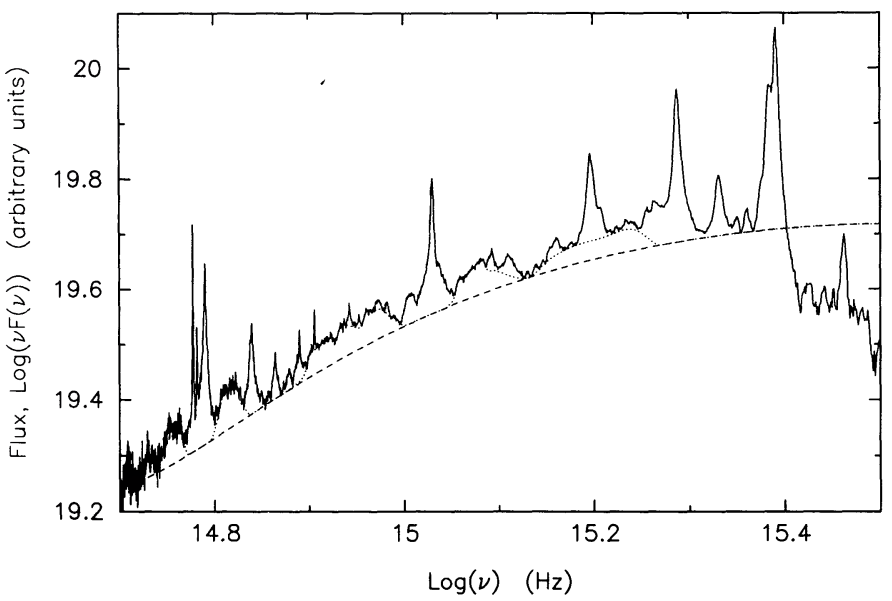

FIG. 6--Logarithmic plot of the composite, $v F(v)$ vs. rest-frame wavelength (solid line), with the continuum (dashed line) and the five $\mathrm{Fe}$ II complex fits (dotted lines).

noted below many of the weaker features seen in Figures 6 and 7 may be peaks in the $\mathrm{Fe}$ II emission.

The large number of components evident in the composite, some of which cover an extended range in wavelength, make the assignment of a "continuum" difficult. Figure 6 shows the composite plotted logarithmically with the "operational" continuum we adopted in order to define and measure the feature equivalent widths. The operational continuum is a cubic spline, fitted through the possible continuum windows at 1285 , 2200,4200 and $5770 \AA$. This allows for the apparent softening of the spectrum in the ultraviolet, while minimizing the deviation of the continuum from a power-law. A single power-law could be fitted to the composite at 1285 and $5770 \AA$ but this fit would then fall far below the composite everywhere between these points. If 1680 or $2650 \AA$ were taken as continuum regions, the height of the composite above the continuum could be reduced, but at the cost of a strongly curved continuum.

Over intermediate-wavelength scales several features contribute significantly: the Balmer continuum radiation produces a relatively smooth feature in the spectrum, and the large number of $\mathrm{Fe}$ II multiplets blend together to produce wavelength regions characterized by a significant excess of flux, with certain multiplets visible as individual emission features.

An attempt has been made to fit the Balmer continuum radiation and the features arising from the "blended" fraction of the principal Fe II complexes. The features have been fitted in five sections using an interactive spline routine from the FIGARO data reduction package. The wavelengths and extent of the sections were determined using a combination of theoretical models (Wills, Netzer, \& Wills 1985) and direct inspection of the composite. The aim of this procedure is to define an "operational" continuum for the definition of emission lines that lie in wavelength regions where Fe II and the Balmer continuum contributions are significant. The fits adopted are shown in Figures 6 and 7. Note that the form of these features is critically affected by the large-scale continuum adopted (in particular the use of $2200 \AA$ as a continuum window), and is particularly uncertain in regions where strong broad emission lines are present - e.g., the $\mathrm{Mg}$ II region. No attempt has been made to incorporate individual $\mathrm{Fe}$ II lines in the fits and many of the weak emission features visible in the regions of the spline fits correspond to individual Fe II multiplets. No attempt was made to fit the Balmer continuum separately from the $\mathrm{Fe}$ II complex, and we are unable to provide any credible estimate of the intensity, effective temperature, or optical depth of the Balmer continuum.

There is a considerable disparity between estimates of emission line equivalent widths in the literature, particularly for the weaker lines, yet the spectra from which the differing estimates


Fig. 7.-Enlargements of the composite, $\lambda F(\lambda)$ vs. rest-frame wavelength (solid line), showing the continuum (dashed line) and Fe II complex fits (dotted lines) together with the identification of features listed in Table 1. 
were obtained do not always appear to show significant differences. Much of the disparity can be ascribed to different "continuum" definitions coupled with varying wavelength ranges employed in the equivalent-width calculations. Using the emission feature wavelength limits-Table 1-together with the form of the continuum shown in Figure 6 it should be possible to identify clearly how the equivalent widths and standard deviations listed in Table 1 have been measured, which should facilitate comparison with spectra from other sources. Figure 7 shows three close-ups of Figure 2, in order to enhance the visibility of weak emission features. Once again the operational continuum fit including the $\mathrm{Fe}$ II/Balmer continuum fits is shown.

\section{DISCUSSION}

There are well-documented luminosity and redshift dependent effects in quasar emission spectra (e.g., Kinney, Rivolo, \& Koratkar 1990; O'Brien, Gondhalekar, \& Wilson 1988), and in interpreting this composite spectrum it is important to realise that there is a strong dependence with rest-frame wavelength of the mean absolute magnitude of the quasars contributing. To emphasize the importance of this point, we show in Figure 8 the mean absolute magnitude of the quasars contributing to the composite as a function of rest-frame wavelength together with the standard deviations of the absolute magnitude distribution. There may also be a dependence of spectral properties on redshift but we are unable to separate any redshift and luminosity dependent effects from the currently available data.

There are four particularly striking features of the composite:

1. The number of weak emission features. Figure 7 reveals a number of low-level emission components, even in supposed “continuum windows" such as $2200-2250 \AA$. In addition, the strong lines have such broad wings that they are all blended to some extent, implying that the operational continuum is an upper limit, and that all line fluxes must be treated with caution.

2. The prominence of the $\mathrm{Fe}$ II emission. We measure a Lyman- $\alpha$ to $\mathrm{Fe}$ II/Balmer continuum ratio of about unity (for $\mathrm{N} v$ at $30 \%$ the strength of Lyman- $\alpha$ ). This is comparable to the value found by Wills et al. (1985), given our use of $2200 \AA$ as a continuum window and their relatively restricted wavelength coverage, and is clearly far above the ratio predicted by standard photoionization models.

3. The continuum, which would be seen as a straight line in Figure 6, is not well represented by a power-law on any but the smallest of scales.

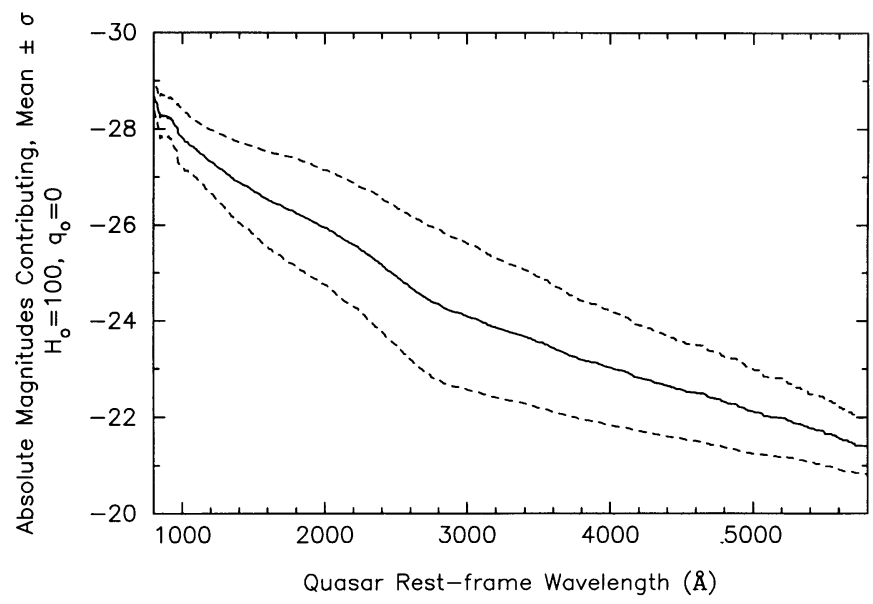

FIG. 8.-Mean absolute magnitude (solid line), and associated $\pm 1 \sigma$ range (dashed lines), of the quasar and AGN population contributing to the composite as a function of rest-frame wavelength

4. Force-fitting a power-law slope to the constituent spectra yields a median spectral index of $\sim-0.3$, significantly harder than the canonical value of -0.7 found by such authors as Richstone \& Schmidt (1980), O'Brien et al. (1988), and Sargent, Steidel, \& Boksenberg (1989), though it agrees with the results from recent multifrequency surveys (Neugebauer et al. 1987; Sanders et al. 1989). Since both O'Brien et al. (1988) and Sargent et al. (1989) made measurements confined to the ultraviolet, the softening of the spectrum in this region may be responsible for at least part of the discrepancy. Finally, the hard excess may be luminosity or redshift-dependent, showing prominently in our high-luminosity sample yet being virtually undetectable in the lower luminosity, nearby active galactic nuclei.

The LBQS is supported by National Science Foundation grants nos. AST 90-01181, 90-05117, 87-00741 and 86-20427, for which we are grateful. The LBQS would not have been possible without the active support of the United Kingdom Schmidt Telescope Unit and the staff of the Automated Plate Measuring facility. We acknowledge the computing support provided by the Starlink Project which is funded by the UK SERC. P. C. H. is pleased to acknowledge the receipt of a Royal Society Research Fellowship. P. J. F. is supported by an SERC postgraduate studentship. F. H. C. wishes to thank the Institute of Astronomy for its hospitality and the Royal Society for support through its Guest Research Fellowship program.

\section{REFERENCES}

Boyle, B. J. 1990, MNRAS, 243, 231

Cristiani, S., \& Vio, R. 1990, A\&A, 227, 385

Foltz, C. B., Chaffee, F. H., Hewett, P. C., MacAlpine, G. M., Turnshek, D. A. Weymann, R. J., \& Anderson, S. F. 1987, AJ, 94, 1423

Foltz, C. B., Chaffee, F. H., Hewett, P. C., Weymann, R. J., Anderson, S. F., \& MacAlpine, G. M. 1989, AJ, 98, 1959

Hewett, P. C., Foltz, C. B., Chaffee, F. H., Francis, P. J., Weymann, R. J., Morris, S. L., Anderson, S. F., \& MacAlpine, G. M. 1991, AJ, in press

Kinney, A. L., Rivolo, A. R., \& Koratkar, A. P. 1990, ApJ, 357, 338
Neugebauer, G., Green, R. F., Matthews, K., Schmidt, M., Soifer, B. T., \& Bennett, J. 1987, ApJS, 63, 615

O'Brien, P. T., Gondhalekar, P. M., \& Wilson, R. 1988, MNRAS, 233, 801

Richstone, D. O., \& Schmidt, M. 1980, ApJ, 235, 361

Sanders, D. B., Phinney, E. S., Neugebauer, G., Soifer, B. T., \& Matthews, K 1989, ApJ, 347, 29

Sargent, W. L., Steidel, C. C., \& Boksenberg, A. 1989, ApJS, 69, 703

Wills, B., Netzer, H., \& Wills, D. 1985, ApJ, 288, 94 